\title{
Donor kidney quality assessment with ultrasound and clinical parameters in deceased donor kidney transplantation
}

\author{
Sang Oh Yun ${ }^{1}$, Kyo Won Lee ${ }^{1}$, Jae Berm Park ${ }^{1}$, Sung Yoon Park ${ }^{2}$ \\ ${ }^{1}$ Department of Surgery-Transplantation, Samsung Medical Center, Seoul, Korea \\ ${ }^{2}$ Department of Radiology, Samsung Medical Center, Seoul, Korea
}

Background: We try to evaluate the effect of functional kidney volume measured by donor kidney ultrasound, alone or in combination with other donor factors and see if those parameters could be a model for deciding acceptable kidney grafts in deceased donor kidney transplantation (KT).

Methods: From 2000 to 2020 who underwent deceased donor KT and those donors were recruited only at the Samsung Medical Center and total 273 patients were enrolled after adjusting exclusion criteria. Estimated glomerular filtration rate (eGFR) was the laboratory lab used for evaluating kidney function. To binarize each kidney US character, we selected an optimal cut-off value for eGFR less than 30 within 1 year after KT by using Youden's index among the values which have specificity above $60 \%$. Cox regression analysis was performed for eGFR less than 30 within 1 year after KT and graft failure within 2 years after KT.

Results: All the US findings were risk factors of eGFR less than 30 within year after KT. Odds ratios of model 3 of renal length, cortical thickness, parenchymal thickness and renal length $\times$ cortical thickness are 6.417 (95\% confidence interval [Cl], $1.332-$ 28.361; $P=0.020), 10.146$ (95\% Cl, 1.934-53.217; $P=0.006), 6.665$ (95\% Cl, 1.540-28.852; $P=0.011)$ and $14.700(95 \% \mathrm{Cl}, 2.805-$ 77.030; $P=0.001)$, respectively. About graft failure within 2 years after $K T$, cortical thickness and renal length $\times$ cortical thickness were risk factors and odd ratios of them are $3.792(95 \% \mathrm{Cl}, 1.006-14.295 ; \mathrm{P}=0.049)$, and 4.129 (95\% Cl, 1.119-15.236; $\mathrm{P}=0.033)$, respectively.

Conclusions: We confirmed that the donor kidney US findings act as predictors of short term prognosis after KT and thought that this can be used to help in deceased donor selection. However, the ultrasound findings alone cannot be a standard, and it is thought to have a meaning as a factor constituting the extended criteria.

Corresponding author: Kyo Won Lee

E-mail:kw1980.lee@gmail.com

(c) The Korean Society for Transplantation
This is an Open Access article distributed under the terms of the Creative Commons Attribution Non-Commercial License (http://creativecommons.org/li-
censes/by-nc/4.0/) which permits unrestricted non-commercial use, distribution, and reproduction in any medium, provided the original work is properly cited. 